\title{
Precision Measurement of Nuclei in Cosmic Rays with the Alpha Magnetic Spectrometer on the International Space Station.
}

\section{A. Oliva* on behalf of the AMS Collaboration.}

Centro de Investigaciones Energéticas, Medioambientales y Tecnológicas (CIEMAT), E-28040

Madrid, Spain.

E-mail: alberto.oliva@cern.ch

\begin{abstract}
AMS-02 is wide acceptance high-energy physics experiment installed on the International Space Station in May 2011 and operating continuously since then. With a collection rate of approximately $1.7 \times 10^{10}$ events/year, and the combined identification capabilities of 5 independent detectors, AMS-02 is able to precisely separate cosmic rays light nuclei $(1 \leq Z \leq 8)$. Knowledge of the precise rigidity dependence of the light nuclei fluxes is important in understanding the origin, acceleration, and propagation of cosmic rays. AMS-02 collaboration has recently released the precise measurements of the proton and helium fluxes as function of rigidity (momentum/charge). The spectral indexes of both fluxes progressively hardens at rigidities larger than $100 \mathrm{GV}$. The rigidity dependence of the helium flux spectral index is similar to that of the proton spectral index though the magnitudes are different. Remarkably, the spectral index of the proton to helium flux ratio increases with rigidity up to $45 \mathrm{GV}$ and then becomes constant; i.e. the flux ratio above 45 GV is well described by a single power law. Preliminary results about the spectral shape of other light nuclei have been shown during the conference and are here briefly introduced.
\end{abstract}

38th International Conference on High Energy Physics

3-10 August 2016

Chicago, USA

${ }^{*}$ Speaker. 


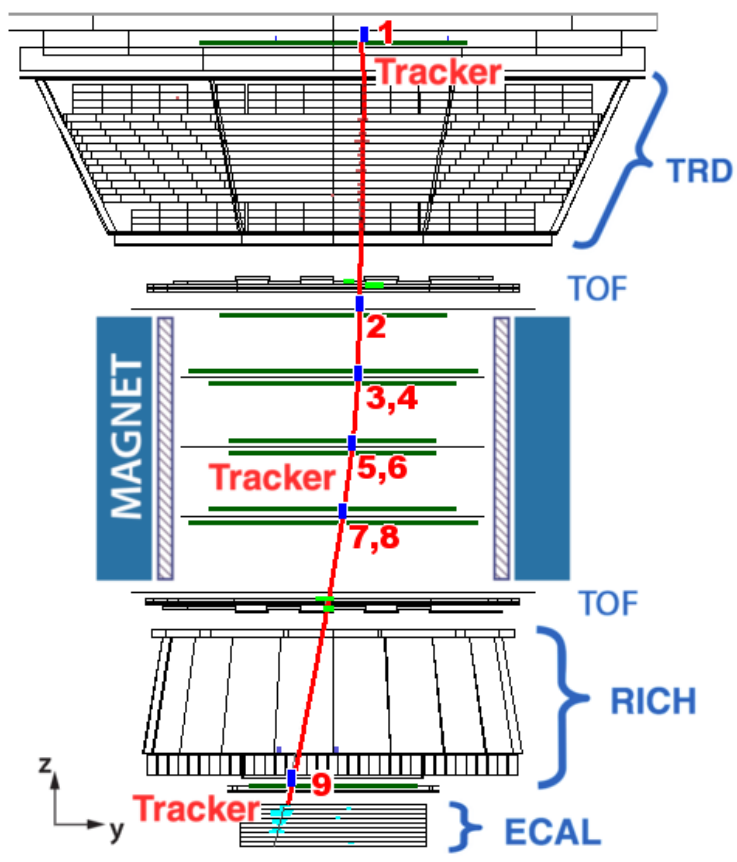

Figure 1: The layout of the AMS-02 detector. It consists of nine planes of precision silicon tracker a transition radiation detector (TRD), four planes of time of flight counters (TOF), a permanent magnet, an array of anticoincidence counters (ACC), surrounding the inner tracker, a ring imaging Čerenkov detector $(\mathrm{RICH})$, and an electromagnetic calorimeter (ECAL). A low energy event traversing the AMS detector is superimposed.

Knowledge of the precise behavior of the nuclei spectra $1 \leq Z \leq 8$ is important in understanding the origin, acceleration, and propagation of cosmic rays [1]. In particular protons and completely ionised helium nuclei are the most abundant charged particles in cosmic rays. The most recent measurements of the proton and the helium fluxes in cosmic rays have reported different variations of the flux with energy $[2,3,4,5]$. These measurements generated widespread interest. In particular, the ATIC-2, CREAM, and PAMELA experiments showed deviations of the proton flux from a single power law. Different scenarios were proposed to account for the hardening of the flux based on different sources, acceleration mechanisms, diffusive propagation effects, and their superposition [6]. In this work we will discuss the recent measurements of AMS of the proton and helium spectra based on data collected by the Alpha Magnetic Spectrometer (AMS) during the first 30 months (May 19, 2011 to November 26, 2013) of operation onboard the International Space Station (ISS) [7, 8].

The Alpha Magnetic Spectrometer (AMS-02) is a general purpose high-energy particle physics detector. It was installed on the International Space Station (ISS) on 19 May 2011 to conduct a unique long duration mission ( $>20$ years) of fundamental physics research in space. The layout of the AMS-02 detector [9] is shown in Fig. 1. The two main detectors used for the nuclei spectra measurement are the tracker and the time-of-flight (TOF). The tracker is composed by 9 layers of silicon double-side micro-strip sensors. There is one layer on top of AMS (L1), 7 layers inside the 
magnet (L2 to L8) constituting the inner tracker, and one layer on the bottom of AMS on top of the calorimeter (L9). The tracker accurately determines the trajectory and absolute charge $|Z|$ of cosmic rays by multiple measurements of the coordinates and energy loss. Together, the tracker and the magnet measure the rigidity $R$ of charged cosmic rays. The spatial resolution in each tracker layer is $10(7.5) \mu \mathrm{m}$ for proton(helium) in the bending direction. With a magnetic field of $1.4 \mathrm{kG}$, the maximum detectable rigidity, i.e. the rigidity for which $\Delta R / R=100 \%$, is $1.8(3.2) \mathrm{TV}$ over the $3 \mathrm{~m}$ lever arm from L1 to L9. Each layer of the tracker also provides an independent measurement of the absolute value of the charge $|Z|$ of the cosmic ray. The charge resolution of the layers of the inner tracker together is $\Delta Z=0.05(0.07)$ for proton(helium). Two planes of TOF counters are located above the magnet, and two planes are located below it. The difference in time between the upper TOF and the lower TOF, together with the track length measurement from tracker, measure the particle velocity $(\beta=v / c)$. The velocity resolution has been measured to be $\Delta \beta / \beta=0.04(0.02) \times \beta$ for $Z=1(2)$ particles. This discriminates between upward- and downward-going particles. The pulse heights of the two upper layers, are combined to provide an independent measurement of the charge. The pulse heights from the two lower planes are combined to provide another independent charge measurement with a similar accuracy. Since launch in May 2011, all the detectors have been monitored constantly and calibrated periodically to ensure steady performances.

AMS simulation is based on the GEANT-4.9.6 package [10]. This program simulates electromagnetic and hadronic interactions of particles in the material of AMS. The simulated detector signals are then digitised according to the measured characteristics of the electronics. Simulated events undergo the same reconstruction of the flight data.

In the first 30 months AMS collected $4.1 \times 10^{10}$ cosmic ray events. The effective data collection time includes only those seconds during which the detector was in normal operating conditions. Events are selected to be down-going and to have a charge compatible $Z=1(2)$ in the tracker and in the TOF for proton(helium) sample. In order to have the best resolution at the highest rigidities, further selections are made by requiring the track to pass through L1 and L9 and to satisfy additional track fitting quality criteria such as a $\chi^{2} /$ n.d.f. $<10$. To select only primary cosmic rays, well above the geomagnetic cutoff, the measured rigidity is required to be greater than 1.2 times the maximum geomagnetic cutoff within the AMS field of view. For the proton sample a small contamination below $2 \mathrm{GeV}$ of pions produced in the upper part of the detector, is removed by requiring that the mass, determined by combining the velocity measured by the TOF with the rigidity, of the particle being larger than $0.5 \mathrm{GeV} / c^{2}$. The background contributions from protons which originated in the interactions of nuclei and at the top of AMS and from $e^{ \pm}$, only noticeable under $2 \mathrm{GV}$, are evaluated with the simulation and subtracted. The helium selected sample contains a small background, less than $10^{-3}$, coming from higher charges nuclei interacting at the top of AMS. The residual background has been evaluated with the simulation and then subtracted. After the background subtraction there are 300 million protons and 50 million helium nuclei in the sample.

The isotropic flux $\Phi_{i}^{Z}$ for the i-th rigidity bin $R_{i}, R_{i}+\Delta R_{i}$ is:

$$
\Phi_{i}^{Z}=\frac{N_{i}^{Z}}{A_{i}^{Z} \varepsilon_{i}^{Z} T_{i} \Delta R_{i}}
$$


where $N_{i}^{Z}$ is the number of events for $Z=1,2$ after background subtraction and corrected for binto-bin rigidity migration, by the use of an unfolding procedure that uses the rigidity resolution function obtained from simulation; $A_{i}^{Z}$ is the effective acceptance calculate with the simulation and corrected for the small differences between data and simulation for reconstruction and selection efficiencies; $\varepsilon_{i}^{Z}$ is the trigger efficiency measured from data with the unbiased trigger events (is $>90 \%$ for both proton and helium); $T_{i}$ is the collection time.

Given the small $(<\%)$ statistical error fluctuation on the number of events, extensive studies were made of the systematic errors. The most important sources of which are: the acceptance calculation, the rigidity resolution function, and the absolute rigidity scale. A detailed discussion of all the systematics can be found in [7, 8].

The systematic error on the effective acceptance accounts for the uncertainties associated to the corrections applied to the acceptance due to the small differences between data and simulation of reconstruction and selection efficiencies. An additional systematic error to the effective acceptance is due to the knowledge of the inelastic interaction in the detector. The inelastic cross sections of protons in AMS material, mostly made of carbon and aluminum, are known with a typical precision of $\sim 10 \%$. By varying the inelastic cross section in the simulation by this amount and calculating the effective acceptance the systematics deviation has been derived. In the case of helium nuclei the inelastic cross section used in the simulation is calculated with the Glauber-Gribov model [10]. The data available in literature for the verification of these cross section are sparse or absent. We then developed a method to measure the survival probability, i.e. the fraction of helium nuclei that do not interact inelastically, in the AMS materials from data to verify the simulation. This method make use of the time periods in which AMS was horizontal with respect to the zenith. In this condition particles traverse AMS from the top to the bottom and from the bottom to the top. With events travelling from L9 to L1 the probability of interaction between L2 and L1 can be calculated selecting events with the inner tracker and measuring the charge on the L1. While using events that traverse AMS from L1 to L9 the survival probability between L8 and L9 can be calculated selecting events with the inner tracker and measuring the charge on L9. From the comparison of these probabilities between data and simulation the cross section model has been validated, and uncertainties estimated. Globally the systematic error associated to acceptance is almost flat with energy and around $1 \%$ for protons, and $2 \%$ for helium.

The systematics associated to the unfolding is mainly due to the uncertainties related to the tracker rigidity resolution function. The tracker rigidity resolution has been measured with high precision at $400 \mathrm{GV}$ using a proton beam extracted from SPS at CERN. These data allowed to verify the absolute rigidity scale, and to verify the rigidity resolution description in the simulation. The tracker resolution function description in the simulation has been also verified using flight data for both proton and helium in two ways. First, the differences of the coordinates measured in L3 or L5 to those obtained from the track fit using the measurements from L1, L2, L4, L6, L7, L8, and L9 were compared between data and simulation, verifying the tracker spatial resolution simulation. Second, the differences between the coordinates measured in L1 and L9 and those obtained from the track fit using the information from only the inner tracker were compared between data and simulation. These comparisons validated the multiple, elastic and quasi-elastic scattering model used in the simulation. From the verifications the uncertainties on the tracker resolution function have been derived, and eventually the corresponding systematics on the number of events after 

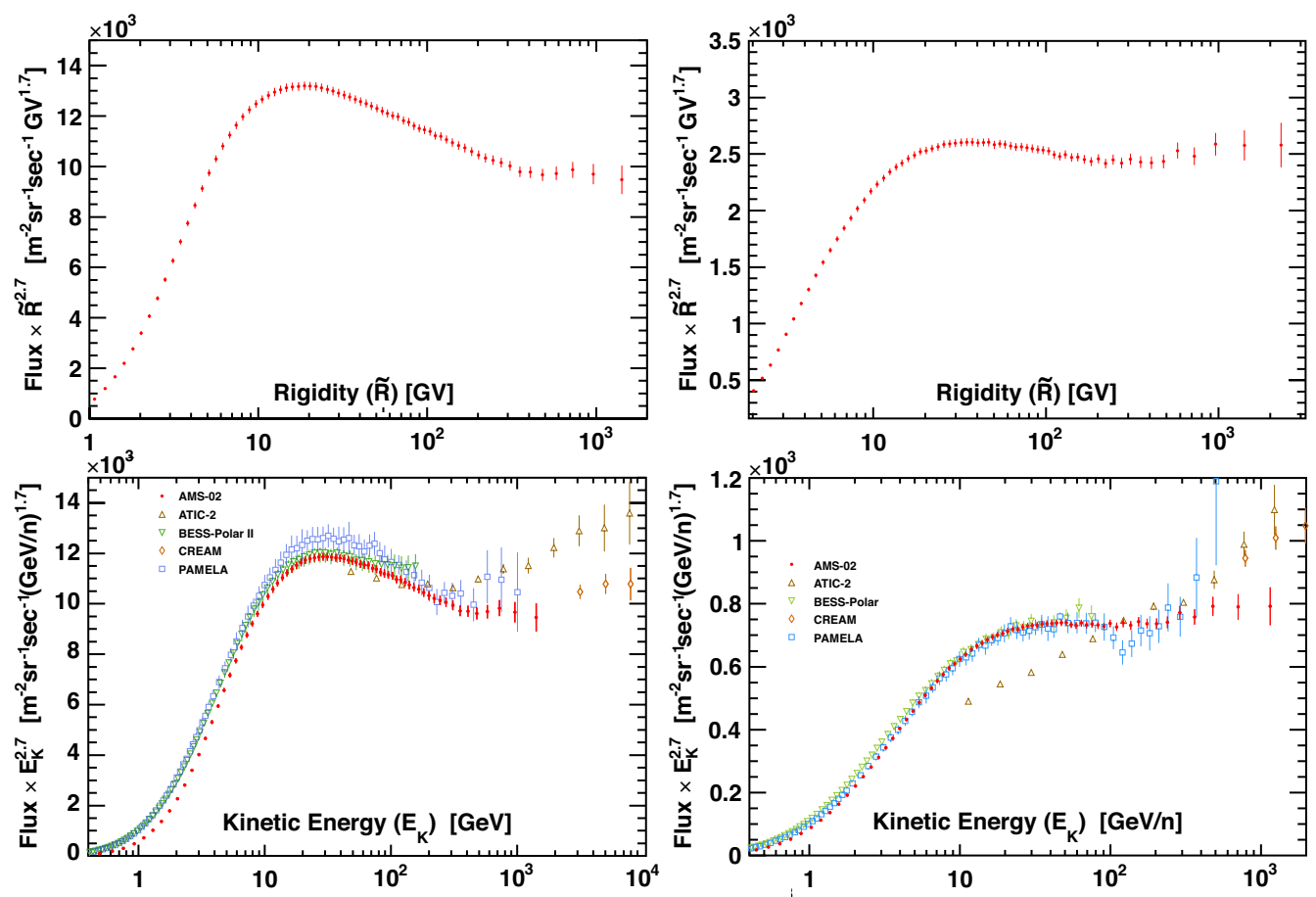

Figure 2: The AMS proton flux (left) and the helium nuclei flux (right) multiplied by $\tilde{R}^{2.7}$ as a function of rigidity $\tilde{R}$ are displayed on the top. On the bottom the proton (left) and the helium (right) fluxes multiplied by $E_{K}^{2.7}$ as function of kinetic energy per nucleon $E_{K}$ compared with recent measurements [2, 3, 4, 5]. For the AMS results we convert the rigidity into kinetic energy using $E_{K}=\left(\sqrt{Z R^{2}+M^{2}}-M^{2}\right) / A$. For $Z=1$ spectrum we assume the sample purely composed by protons $\left(A=1, M=0.938 \mathrm{GeV} / c^{2}\right)$, while $Z=2$ was treated as containing only ${ }^{4} \mathrm{He}\left(A=2, M=3.726 \mathrm{GeV} / c^{2}\right)$.

unfolding calculated. The systematic error associated to unfolding has been found to be less than $1 \%$ below 200(400) GV and 3(3.5)\% at 1.8(3) TV for proton(helium).

The main contribution to to the systematic uncertainty on the rigidity scale is due to residual tracker misalignment. This has been estimated with flight data by comparing the $E / p$ ratio for electron and positron events, where $E$ is the energy measured with the ECAL and $p$ is the momentum measured with the tracker. It was found to be $1 / 26 \mathrm{TV}^{-1}$, limited by the 30 months high energy positron statistics.

Heavier nuclei analysis follows similar lines the one of proton and helium. The most important difference are: the lower statistics available for higher charges; the background contamination of interactions of heavier nuclei that is more important; the cross sections of interactions of nuclei in material are less known an their effect is larger. These analysis, and their preliminary results, have been shown during the conference.

Figure 2(top) shows the proton and helium fluxes as a function of rigidity with the total errors. In these and the subsequent figures, the points are placed along the abscissa at $\tilde{R}$ calculated for a flux $\propto R^{-2.7}$. Figure 2(bottom) shows the AMS flux as a function of kinetic energy per nucleon $E_{K}$ together with the most recent results (i.e., from experiments after the year 2000). 


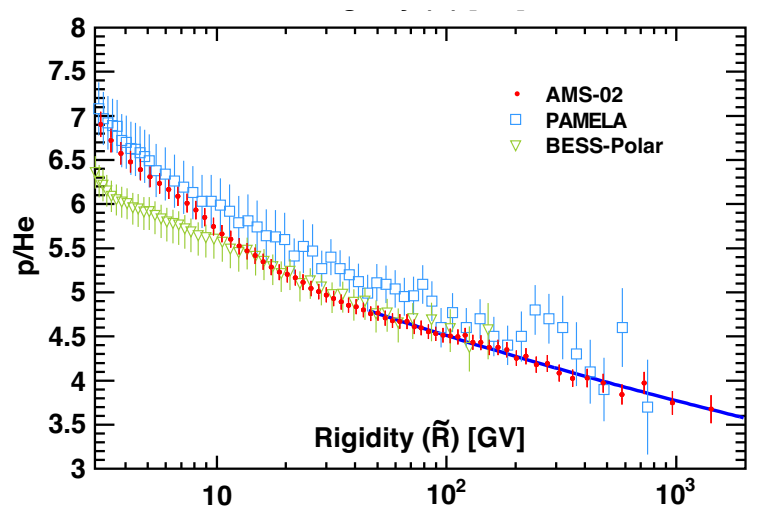

Figure 3: The $p / \mathrm{He}$ ratio as a function of rigidity compared with recent measurements $[3,5]$. The solid blue curve indicates the fit of a single power law $\left(\propto R^{\gamma}\right)$ to the AMS data. As seen, above $45 \mathrm{GV}$ the ratio is well described by a single power law.

A power law with a constant spectral index $\gamma, \Phi=C R^{\gamma}$, where $R$ is in $\mathrm{GV}$ and $C$ is a normalization factor, does not provide a good description of the AMS proton flux or of the AMS helium flux. A better description of the flux is obtained using a double power law function. The fit is performed starting from $45 \mathrm{GV}$ to minimise the effect of solar modulation. The fitting procedure and the detailed results are described in $[7,8]$. The double power law description give a compatible position for the spectral shape turning point $R^{0}$ : for proton $R_{p}^{0}=336 \pm 95$ and for helium nuclei $R_{\mathrm{He}}^{0}=245 \pm 48$. Also similar is the amount of spectral variation $\Delta \gamma, \Delta \gamma_{p}=0.133 \pm 0.056$ and $\Delta \gamma_{\mathrm{He}}=0.119 \pm 0.036$. A significative difference in the spectral shape is instead observed for the spectral index absolute value $\gamma$, that are $\gamma_{p}=-2.849 \pm 0.006$ and $\gamma_{\mathrm{He}}=-2.780 \pm 0.006$.

To better examine the difference between the rigidity dependence of the proton and helium fluxes, the ratio of the proton flux to the helium flux, or $p /$ He ratio can be calculated taking in proper account the correlation between the systematic errors of the unfolding and of the absolute rigidity scale. Figure 3 shows the AMS $p / \mathrm{He}$ ratio. Above $45 \mathrm{GV}$ the $p / \mathrm{He}$ ratio is well fit with a single power law with a spectral index of $\gamma_{p / \mathrm{He}}=-0.077 \pm 0.007$.

In conclusion the knowledge of the precise behavior of the nuclei spectra $1 \leq Z \leq 8$ is important in understanding the origin, acceleration, and propagation of cosmic rays. The AMS measurement of proton and helium fluxes based on 300 million and 50 millions events respectively showed that both fluxes deviates from a single power law and that both spectral indexes progressively hardens at rigidities larger than $100 \mathrm{GV}$. The magnitude of the helium spectral index is different from that of the proton spectral index, but the rigidity dependence is similar for helium and protons. The proton to helium flux ratio spectral index increases with rigidity up to about $45 \mathrm{GV}$ and becomes constant for higher rigidities, i.e. the proton to helium flux ratio is well described by a single power law above $45 \mathrm{GV}$. Progress in the analysis of the other light nuclei species have been discussed, and preliminary results showed during the conference.

The author would like to acknowledge CIEMAT, CDTI, and SEIDI-MINECO, Spain [grant numbers AYA2012-39526-C02-(01/02), ESP2015-71662-C2-(1-P/2-P), SEV-2011-0187, SEV-20150548, and MDM-2015-0509]. 


\section{References}

[1] See for example: I. A. Grenier, J. H. Black and A. W. Strong, Annu. Rev. Astron. Astrophys. 53, 199 (2015); P. Blasi, Astron. Astrophys. Rev. 21, 70 (2013); A. W. Strong, I. V. Moskalenko, and V. S. Ptuskin, Annu. Rev. Nucl. Part. Sci. 57, 285 (2007).

[2] A. D. Panov et al., Bull. Russ. Acad. Sci. Phys. 73, 564 (2009), extracted from D. Maurin, F. Melot, and R. Taillet, Astron. Astrophys. 569, A32 (2014).

[3] T. Sanuki et al., Astrophys. J. 545, 1135 (2000); K. Abe et al., Astrophys. J. 822, 65 (2016).

[4] Y. S. Yoon et al., Astrophys. J. 728, 122 (2011);

[5] O. Adriani et al., Astrophys. J. 765, 91 (2013); O. Adriani et al., Science 332, 69 (2011).

[6] See for example: N. Tomassetti, Phys. Rev. D 92, 081301(R) (2015); S. Thoudam and J. R. Hörandel, Astronom. Astrophys. 567, A33 (2014); A. E. Vladimirov, G. Jóhannesson, I. V. Moskalenko, and T. A. Porter, Astrophys. J. 752, 68 (2012).

[7] M. Aguilar et al., Phys. Rev. Lett. 114, 171103 (2015).

[8] M. Aguilar et al., Phys. Rev. Lett. 115, 211101 (2015).

[9] A. Kounine, Int. J. Mod. Phys. E 211230005 (2012);

[10] J. Allison et al., IEEE Trans. Nucl. Sci. 53, 270 (2006); S. Agostinelli et al., Nucl. Instrum. Methods Phys. Res., Sect. A 506, 250 (2003). 